\title{
Asymptotic equivalence of a quasilinear impulsive differential equation and a linear ordinary differential equation
}

M. U. Akhmet and M. A. Tleubergenova 


\title{
ASYMPTOTIC EQUIVALENCE OF A QUASILINEAR IMPULSIVE DIFFERENTIAL EQUATION AND A LINEAR ORDINARY DIFFERENTIAL EQUATION
}

\author{
M. U. AKHMET AND M. A. TLEUBERGENOVA
}

Received 17 October, 2007

\begin{abstract}
The main goal of the paper is to obtain new sufficient conditions for the asymptotic equivalence of the quasilinear impulsive system of differential equations and the linear system of ordinary differential equations.
\end{abstract}

1991 Mathematics Subject Classification: Primary 34A37; Secondary 34C41, 34D05

Keywords: asymptotic equivalence, quasilinear systems, impulsive differential equations

\section{INTRODUCTION AND PRELIMINARIES}

As it is well known, one of the most efficient ways to clarify the structure of a complex set is to arrange an equivalence with another set which is then carefully analysed. Apparently, the first results concerning the asymptotic behaviour of systems on the basis of one-to-one correspondence between sets of solutions were obtained in $[4,5,8,9]$.

In this paper, we obtain new sufficient conditions for the asymptotic equivalence of linear and quasilinear systems of differential equations with piecewise constant arguments. Homeomorphism, which is the most important version of the correspondence in a theoretical sense, is considered. The method of the paper was also used in the works $[1,2]$.

Let $\mathbb{Z}, \mathbb{N}$, and $\mathbb{R}$ be, respectively, the sets of all integer, natural, and real numbers. Denote by $\|\cdot\|$ the Euclidean norm in $\mathbb{R}^{n}$, where $n \in \mathbb{N}$. Fix a sequence $\theta_{i}, i \in \mathbb{Z}$, such that $\theta_{i}<\theta_{i+1}$ for all $i \in \mathbb{Z}$ and $\left|\theta_{i}\right| \rightarrow \infty$ as $|i| \rightarrow \infty$.

We consider the system of impulsive differential equations

$$
\begin{gathered}
z^{\prime}(t)=C z(t)+f(t, z(t)), \\
\left.\Delta z\right|_{t=\theta_{i}}=J_{i}(z),
\end{gathered}
$$

and the linear system of ordinary differential equations

$$
x^{\prime}(t)=C x(t),
$$


where $x, z \in \mathbb{R}^{n}, t \in \mathbb{R}$.

The following assumptions will be needed throughout the paper:

(c1) $C$ is a constant $n \times n$ real valued matrix;

(c2) $f \in C\left(\mathbb{R} \times \mathbb{R}^{n}, \mathbb{R}^{n}\right)$;

(c3) The estimate

$$
\left\|f\left(t, z_{1}\right)-f\left(t, z_{2}\right)\right\|+\left\|J_{i}\left(z_{1}\right)-J_{i}\left(z_{2}\right)\right\| \leq \eta(t)\left\|z_{1}-z_{2}\right\|
$$

holds for all $z_{1}, z_{2} \in \mathbb{R}^{n}, t \in \mathbb{R}_{+}$, and $i \in \mathbb{Z}$ with some nonnegative function $\eta(t)$ defined on $\mathbb{R}_{+}$;

(c4) The following equalities are true

$$
\begin{aligned}
f(t, 0)=0 & \text { for all } t \in \mathbb{R} ; \\
J_{i}(0)=0 & \text { for all } i \in \mathbb{Z} .
\end{aligned}
$$

To investigate the asymptotic properties of the solutions, the following definition can be efficiently applied.

Definition ([3,6]). A homeomorphism $H$ between the sets of solutions $x(t)$ and $z(t)$ is called an asymptotic equivalence if $z(t)=H(x(t))$ implies that $x(t)-z(t) \rightarrow$ 0 as $t \rightarrow \infty$.

\section{MAIN RESULT}

In this section we consider the theorem about the asymptotic equivalence of systems (1) and (2). The theorem is a development of V. Yakubovich's result [6,9] for impulsive differential equations. Let $\alpha=\min _{j} \operatorname{Re} \lambda_{j}$ and $\beta=\max _{j} \operatorname{Re} \lambda_{j}$, where $\operatorname{Re} \lambda_{j}$ denotes the real part of the eigenvalue $\lambda_{j}$ of the matrix $C$. Let $m_{\alpha}$ and $m_{\beta}$ be the maximum of the degrees of elementary divisors of $C$ corresponding to eigenvalues with the real parts equal to $\alpha$ and $\beta$, respectively. Clearly, there exist constants $\kappa_{1}$ and $\kappa_{2}$ such that

$$
\left\|e^{C t}\right\| \leq \kappa_{1} t^{m_{\beta}-1} e^{\beta t} \text { and }\left\|e^{-C t}\right\| \leq \kappa_{2} t^{m_{\alpha}-1} e^{-\alpha t}
$$

for all $t \in \mathbb{R}_{+}=[0, \infty)$. We shall also assume that

(c5) The relation

$$
l_{0}:=\int_{0}^{\infty} t^{m_{\beta}+m_{\alpha}-2} e^{(\beta-\alpha) t} \eta(t) d t+\sum_{0 \leq \theta_{i}<\infty} \theta_{i}^{m_{\beta}+m_{\alpha}-2} e^{(\beta-\alpha) \theta_{i}} \eta\left(\theta_{i}\right)<+\infty
$$

is satisified.

The following lemma can be easily established by direct substitution.

Lemma 1. If $z(t)$ is a solution of (1), then there is a solution $u(t)$ of the equation

$$
\begin{gathered}
u^{\prime}=e^{-C t} f\left(t, e^{C t} u\right), \\
\left.\Delta u\right|_{t=\theta_{i}}=e^{-C \theta_{i}} J_{i}\left(e^{C \theta_{i}} u\right),
\end{gathered}
$$


such that

$$
z(t)=e^{C t} u(t) .
$$

Conversely, if $u(t)$ is a solution of (4), then $y(t)$ in (5) is a solution of (2).

Lemma 2. If conditions (c1)-(c5) are valid, then every solution of (4) is bounded on $\mathbb{R}_{+}$and for each solution $u$ of (4) there exists a constant vector $c_{u} \in \mathbb{R}^{n}$ such that $u(t) \rightarrow c_{u}$ as $t \rightarrow \infty$.

Proof. Let $u(t)=u\left(t, t_{0}, u_{0}\right)$ denote a solution of (4) satisfying $u\left(t_{0}\right)=u_{0}, t_{0} \geq 0$. It is known [7] that

$$
u(t)=u_{0}+\int_{t_{0}}^{t} e^{-C s} f\left(s, e^{C s} u(s)\right) d s+\sum_{t_{0} \leq \theta_{i}<t} e^{-C \theta_{i}} J_{i}\left(e^{C \theta_{i}} u\right)
$$

for all $t \geq t_{0}$. Using (c4), we obtain

$$
\begin{aligned}
\|u(t)\| \leq\left\|u_{0}\right\|+k_{1} \int_{t_{0}}^{t} s^{m_{\beta}+m_{\alpha}-2} e^{(\beta s-\alpha) s} \eta(s)\|u(s)\| d s & \\
& +k_{2} \sum_{t_{0} \leq \theta_{i}<t} \theta_{i}^{m_{\beta}+m_{\alpha}-2} e^{(\beta-\alpha) \theta_{i}} \eta\left(\theta_{i}\right)\left\|u\left(\theta_{i}\right)\right\|, \quad t \geq t_{0},
\end{aligned}
$$

with some positive $k_{1}$ and $k_{2}$. By applying the Gronwall-Bellman Lemma for discontinuous functions [7] we obtain that $|u(t)| \leq M$ for all $t \in \mathbb{R}_{+}$, with some positive $M$. To prove the second part of the theorem, we first note that

$$
\begin{gathered}
\left|\int_{t_{0}}^{t} e^{-C s} f\left(s, e^{C s} u(s)\right) d s+\sum_{t_{0} \leq \theta_{i}<t} e^{-C \theta_{i}} J_{i}\left(e^{C \theta_{i}} u\left(\theta_{i}\right)\right)\right| \\
\leq M k_{1} \int_{0}^{\infty} t^{m_{\beta}+m_{\alpha}-2} e^{(\beta-\alpha) t} \eta(t) d t \\
+M k_{2} \sum_{0 \leq \theta_{i}<\infty} \theta_{i}^{m_{\beta}+m_{\alpha}-2} e^{(\beta-\alpha) \theta_{i}} \eta\left(\theta_{i}\right)<\infty .
\end{gathered}
$$

So we may define

$$
c_{u}=u_{0}+\int_{t_{0}}^{\infty} e^{-C s} f\left(s, e^{C s} u(s)\right) d s+\sum_{t_{0} \leq \theta_{i}<\infty} e^{-C \theta_{i}} J_{i}\left(e^{C \theta_{i}} u\left(\theta_{i}\right)\right) .
$$

It follows that

$$
u(t)=c_{u}-\int_{t}^{\infty} e^{-C s} f\left(s, e^{C s} u(s)\right) d s-\sum_{t \leq \theta_{i}<\infty} e^{-C \theta_{i}} J_{i}\left(e^{C \theta_{i}} u\left(\theta_{i}\right)\right),
$$

which completes the proof. 
Theorem 1. If conditions (c1)-(c5) are satisfied, then every solution $y(t)$ of (1) possesses an asymptotic representation of the form

$$
z(t)=e^{C t}[c+o(1)],
$$

where $c \in \mathbb{R}^{n}$ is a constant vector and for a solution $u(t)$ of (4),

$$
o(1)=-\int_{t}^{\infty} e^{-C s} f\left(s, e^{C s} u(s)\right) d s-\sum_{t \leq \theta_{i}<\infty} e^{-C \theta_{i}} J_{i}\left(e^{C \theta_{i}} u\left(\theta_{i}\right)\right)
$$

The proof follows from Lemma 1 and Lemma 2.

Theorem 2. Assume that conditions (c1)-(c5) are fulfilled, and, moreover,

$$
\begin{aligned}
\lim _{t \rightarrow \infty}\left(\int_{t}^{\infty}(s-t)^{m_{\alpha}-1} s^{m_{\beta}-1} e^{\alpha(t-s)} e^{\beta s} \eta(s) d s\right. & \\
& \left.+\sum_{t \leq \theta_{i}<\infty}\left(\theta_{i}-t\right)^{m_{\alpha}-1} \theta_{i}^{m_{\beta}-1} e^{\alpha\left(t-\theta_{i}\right)} e^{\beta \theta_{i}} \eta\left(\theta_{i}\right)\right)=0 .
\end{aligned}
$$

Then systems (1) and (2) are asymptotically equivalent.

Proof. In view of Lemma 2, we see that

$$
\begin{aligned}
z(t) & =e^{C t}\left(c_{u}-\int_{t}^{\infty} e^{-C s} f\left(s, e^{C s} u(s)\right) d s-\sum_{t \leq \theta_{i}<\infty} e^{-C \theta_{i}} J_{i}\left(e^{C \theta_{i}} u\left(\theta_{i}\right)\right)\right) \\
& =x(t)-\int_{t}^{\infty} e^{-C(s-t)} f\left(s, e^{C s} u(s)\right) d s-\sum_{t \leq \theta_{i}<\infty} e^{-C\left(\theta_{i}-t\right)} J_{i}\left(e^{C \theta_{i}} u\left(\theta_{i}\right)\right),
\end{aligned}
$$

where $x(t)=e^{C t} c_{u}$ is a solution of (2) and $u(t)=u\left(t, t_{0}, u_{0}\right)$ is a solution of (4). It is clear that a given $u_{0}$ results in a homeomorphism between $x(t)$ and $y(t)$. Thus, we find that there exists a bicontinuous and one-to-one correspondence between $u_{0}$ and $c_{u}$. In view of (6), we also see that $x(t)-z(t) \rightarrow 0$ as $t \rightarrow \infty$, which completes the proof of the theorem.

\section{REFERENCES}

[1] M. U. Akhmet and M. A. Tleubergenova, "On asymptotic equivalence of impulsive linear homogenous differential systems," Mat. Zh., vol. 2, no. 2(4), pp. 15-18 (electronic), 2002.

[2] M. U. Akhmet, M. A. Tleubergenova, and A. Zafer, "Asymptotic equivalence of differential equations and asymptotically almost periodic solutions," Nonlinear Anal., vol. 67, no. 6, pp. 1870-1877, 2007.

[3] L. Cesari, Asymptotic behavior and stability problems in ordinary differential equations, ser. Second edition. Ergebnisse der Mathematik und ihrer Grenzgebiete, N. F., Bd. 16. Publishers, New York: Academic Press Inc., 1963.

[4] P. Hartman, Ordinary differential equations. New York: John Wiley \& Sons Inc., 1964. 
[5] P. Hartman and A. Wintner, "Asymptotic integrations of ordinary non-linear differential equations," Amer. J. Math., vol. 77, pp. 692-724, 1955.

[6] V. V. Nemytskii and V. V. Stepanov, Qualitative theory of differential equations, ser. Princeton Mathematical Series, No. 22. Princeton, N.J.: Princeton University Press, 1960.

[7] A. M. Samoillenko and N. A. Perestyuk, Impulsive differential equations, ser. World Scientific Series on Nonlinear Science. Series A: Monographs and Treatises. River Edge, NJ: World Scientific Publishing Co. Inc., 1995, vol. 14, with a preface by Yu. A. Mitropol'skiŭ and a supplement by S. I. Trofimchuk, Translated from the Russian by Yu. Chapovsky.

[8] A. Wintner, "Linear variations of constants," Amer. J. Math., vol. 68, pp. 185-213, 1946.

[9] V. A. Yakubovič, "On the asymptotic behavior of the solutions of a system of differential equations," Mat. Sbornik N.S., vol. 28(70), pp. 217-240, 1951.

\section{Authors' addresses}

M. U. Akhmet

Middle East Technical University, Department of Mathematics and Institute of Applied Mathematics, 06531 Ankara, Turkey

E-mail address: marat@metu.edu.tr

\section{A. Tleubergenova}

Aktobe State Pedagogical University, Department of Mathematics, 34 Moldagulovoi Ave., 463000 Aktobe, Kazakhstan

E-mail address: madina_1970@mail.ru 